International Journal of Pure and Applied Mathematics

Volume 86 No. 4 2013, 621-631

ISSN: 1311-8080 (printed version); ISSN: 1314-3395 (on-line version)

url: http://www.ijpam.eu

doi: http://dx.doi.org/10.12732/ijpam.v86i4.3

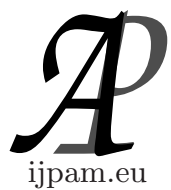

\title{
HYERS-ULAM-RASSIAS STABILITY OF FUNCTIONAL EQUATIONS IN MULTI BANACH SPACES
}

\author{
Ashish $^{1}$, Renu Chugh ${ }^{2}$, Manoj Kumar ${ }^{3} \S$ \\ 1,2,3 Department of Mathematics \\ Maharshi Dayanand University \\ Rohtak, 124001, INDIA
}

\begin{abstract}
In this paper, we study the generalized Hyers-Ulam-Rassias stability of the additive functional equations $f(x+y+z+a)=f(x)+f(y)+f(z)$ for the mapping $f$ from a linear space into a multi-Banach space. Furthermore, we also establish the asymptotic behavior of the above additive functional equations.
\end{abstract}

AMS Subject Classification: 39B82, 39B52, 39B72

Key Words: stability, multi-Banach spaces, additive functional equations

\section{Introduction}

A famous talk presented by Stanislaw M. Ulam [16] in 1940, triggered the study of stability problem for various functional equations. He presented a number of important unsolved problems. One of the interesting problem in the theory of non-linear analysis concerning the stability of homomorphism was as follows: Let $G_{1}$ be a group and let $G_{2}$ be a metric group with the metric $d(\cdot, \cdot)$. Given $\epsilon>0$, does there exist a $\delta>0$ such that if a mapping $h: G_{1} \rightarrow G_{2}$ satisfies the inequality $d(h(x y), h(x) h(y))<\delta$, for all $x, y \in G_{1}$, then there is a homomorphism $H: G_{1} \rightarrow G_{2}$ with $d(h(x), H(x))<\epsilon$, for all $x \in G_{1}$ ?

Received: December 12, 2012

(c) 2013 Academic Publications, Ltd.

${ }^{\S}$ Correspondence author url: www.acadpubl.eu 
If the answer is affirmative, we would say that equation of homomorphism $H(x y)=H(x) H(y)$ is stable.

In 1941, D.H. Hyers [1] was the first mathematician to present the result concerning the stability of functional equations on Banach spaces. This result of Hyers [1] is stated as follows: Let $f: X \rightarrow Y$ satisfies $\|f(x+y)-f(x)-f(y)\| \leq \epsilon$ for all $x, y \in X$ and $\epsilon \geq 0$. Then there exists a unique additive mapping $T: X \rightarrow Y$ such that $\|f(x)-T(x)\| \leq \epsilon$, for all $x \in X$. The generalized version of D.H. Hyers [1] result was given by famous Greece mathematician Th.M. Rassias [20] in 1978, where $f: X \rightarrow Y$ satisfies the inequality $\| f(x+$ $y)-f(x)-f(y) \| \leq \theta\left(\|x\|^{p}+\|y\|^{p}\right)$ for all $x, y \in X$, for some $\theta \geq 0$ and $0 \leq p<1$. The stability paper [21] given by Th.M. Rassias has significantly influenced in the development of stability of functional equations and hence named as Hyers-Ulam-Rassias stability of functional equations. Further, in 1994, P. Gavruta [13] provided a further generalization in which he replaced the bound $\theta\left(\|x\|^{p}+\|y\|^{p}\right)$ by a general function $\phi(x, y)$ for the existence of unique linear mapping. During the last three decades several stability results have been introduced by many famous mathematicians one may also refer to $[2,12,17,18,19,22]$.

In 2007, H.G. Dales and M.S. Moslehian [4] first gave the stability result of Cauchy functional equations on multi-normed spaces and also present some examples on multi-normed spaces. M.S. Moslehian et al. [8] demonstrated the asymptotic aspects of the quadratic functional equations in multi-normed spaces. Further, for a detailed analysis of stability of functional equations in multi-normed spaces one may also refer to $[6,7,10,11]$. The functional equation

$$
f(x+y+z)=f(x)+f(y)+f(z)
$$

is called additive functional equation in 3-variables. The Hyers-Ulam-Rassias stability of this equation was introduced by J.R. Lee and C. Park in 2009 [5] on Banach algebras. The functional equation

$$
f(x+y+z+a)=f(x)+f(y)+f(z)
$$

is called generalized additive functional equation at $a=0$ equation (1.2) reduces into (1.1). Recently, R. Chugh and Ashish [14] introduced the functional equation (1.2) and established the Hyers-Ulam-Rassias stability of this equation. In this paper, using the ideas from [3], we investigate the stability of functional equation (1.2) for the mapping $f$ from a linear space in to multi-Banach spaces. We then establish an asymptotic behavior of additive functional equation (1.2) in multi-Banach spaces. 
Section 2, contains basic definitions and concepts that will be used in the further sections of paper. In the last section, we give the stability of functional equation (1.2) in the sense of multi-Banach spaces.

\section{Preliminaries}

In this section, we adopt some definitions and preliminaries of multi-Banach spaces in the sense of H.G. Dales and M.E. Polyakov [3].

Let $(E,\|\cdot\|)$ be a complex normed space, and let $k \in \mathbb{N}$. We denote by $E^{k}$ the linear space $E \oplus \ldots \oplus E$ consisting of $k$-tuples $\left(x_{1}, x_{2}, \ldots, x_{k}\right)$, where $\left(x_{1}, x_{2}, \ldots, x_{k}\right) \in E$. The linear operations on $E^{k}$ are defined coordinatewise. The zero element of either $E$ or $E^{k}$ is denoted by 0 . We denote by $\mathbb{N}_{k}$ the set $\{1,2, \ldots, k\}$ and by $S_{k}$ the group of permutations on $k$ symbols.

Definition 2.1. A multi-norm on $\left\{E^{k}: k \in \mathbb{N}\right\}$ is a sequence $\left(\|\cdot\|_{k}\right)=$ $\left(\|\cdot\|_{k}: k \in \mathbb{N}\right)$ such that $\|\cdot\|_{k}$ is a norm on $E^{k}$ for each $k \in \mathbb{N},\|x\|_{1}=\|x\|$ for each $x \in E$, and the following axioms are satisfied for each $k \in \mathbb{N}$ with $k \geq 2$ :

(N1) $\left\|\left(x_{\sigma(1)}, x_{\sigma(2)}, \ldots, x_{\sigma(k)}\right)\right\|_{k}=\left\|\left(x_{1}, x_{2}, \ldots, x_{k}\right)\right\|_{k}$

for $\sigma \in S_{k}, x_{1}, x_{2}, \ldots, x_{k} \in E$

(N2) $\left\|\left(\alpha_{1} x_{1}, \alpha_{2} x_{2} \ldots, \alpha_{k} x_{k}\right)\right\|_{k} \leq\left(\max _{i \in \mathbb{N}_{k}}\left|\alpha_{i}\right|\right)\left\|\left(x_{1}, x_{2}, \ldots, x_{k}\right)\right\|_{k}$

for $\alpha_{1}, \alpha_{2}, \ldots, \alpha_{k} \in \mathbb{C}, x_{1}, x_{2}, \ldots, x_{k} \in E$

(N3) $\left\|\left(x_{1}, x_{2} \ldots, x_{k-1}, 0\right)\right\|_{k}=\left\|\left(x_{1}, x_{2}, \ldots, x_{k-1}\right)\right\|_{k-1}$,

for $x_{1}, x_{2}, \ldots, x_{k-1} \in E$

(N4) $\left\|\left(x_{1}, x_{2}, \ldots, x_{k-1}, x_{k-1}\right)\right\|_{k}=\left\|\left(x_{1}, x_{2}, \ldots, x_{k-1}\right)\right\|_{k-1}$

for $x_{1}, x_{2}, \ldots, x_{k-1} \in E$

In this case, we say that $\left(\left(E^{k},\|\cdot\|_{k}\right): k \in \mathbb{N}\right)$ is a multi-normed space (see [3], $[4])$.

Suppose that $\left(\left(E^{k},\|\cdot\|_{k}\right): k \in \mathbb{N}\right)$ is a multi-normed space, and take $k \in \mathbb{N}$. We need the following two properties of multi-norms. They can be found in [3].

(a) $\left\|\left(x_{1}, x_{2}, \ldots, x_{k}\right)\right\|_{k}=\|x\|$, for $x \in E$

(b) $\max _{i \in \mathbb{N}_{k}}\left\|x_{i}\right\| \leq\left\|\left(x_{1}, x_{2}, \ldots, x_{k}\right)\right\|_{k} \leq \sum_{i=1}^{k}\left\|x_{i}\right\| \leq k \cdot \max _{i \in \mathbb{N}_{k}}\left\|x_{i}\right\|$,

for $x_{1}, x_{2}, \ldots, x_{k} \in E$ 
It follows from $(\mathrm{b})$ that if $(E,\|\cdot\|)$ is a Banach space, then $\left(E^{k},\|\cdot\|_{k}\right)$ is a Banach space for each $k \in \mathbb{N}$; in this case, $\left(\left(E^{k},\|\cdot\|_{k}\right): k \in \mathbb{N}\right)$ is a multi-Banach space.

Lemma 2.2. Suppose that $k \in \mathbb{N}$ and $\left(x_{1}, x_{2}, \ldots, x_{k}\right) \in E^{k}$. For each $j=(1,2, \ldots, k)$, let $\left(x_{n}^{j}\right)_{n=1,2, \ldots}$ be a sequence in $E$ such that $\lim _{n \rightarrow \infty} x_{n}^{j}=x_{j}$. Then

$$
\lim _{n \rightarrow \infty}\left(x_{n}^{1}-y_{1}, x_{n}^{2}-y_{2}, \ldots, x_{n}^{k}-y_{k}\right)=\left(x_{1}-y_{1}, x_{1}-y_{1}, \ldots, x_{k}-y_{k}\right)
$$

holds for all $\left(y_{1}, y_{2}, \ldots, y_{k}\right) \in E^{k}$ (see [3], [4]).

Definition 2.3. Let $\left(\left(E^{k},\|\cdot\|_{k}\right): k \in \mathbb{N}\right)$ be a multi-normed space. A sequence $x_{n}$ in $E$ is a multi-null sequence if for each $\epsilon>0$, there exists $n_{0} \in \mathbb{N}$ such that

$$
\sup _{k \in \mathbb{N}}\left\|x_{n}, \ldots, x_{n+k-1}\right\|_{k} \leq \epsilon \quad\left(n \geq n_{0}\right) .
$$

Let $x \in E$, we say that the sequence $\left\{x_{n}\right\}$ is multi-convergent to $x$ in $E$ and write $\lim _{n \rightarrow \infty} x_{n}=x$ if $\left(x_{n}-x\right)$ is a multi-null sequence (see [3], [4]).

Throughout this paper, $a$ is a fixed real number. For convenience, we use the following notation for a given $a \in \mathbb{R} /\{0\}$ and a mapping $f: E \rightarrow F$, from linear spaces into multi-Banach spaces

$$
D_{a}(f(x, y, z))=f(x+y+z+a)-f(x)-f(y)-f(z)
$$

for all $x, y, z \in E$.

\section{Main Results}

In this section, we prove the Hyers-Ulam-Rassias stability of the additive functional equations (2.1).

Theorem 3.1. Let $E$ be a normed space and let $\left(F^{n},\|\cdot\|_{n}\right), n \in \mathbb{N}$ be a multi-Banach space. Let $f: E \rightarrow F$ be a mapping such that

$$
\sup _{k \in \mathbb{N}}\left\|D_{a}\left(f\left(x_{1}, y_{1}, z_{1}\right)\right), D_{a}\left(f\left(x_{2}, y_{2}, z_{2}\right)\right), \ldots, D_{a}\left(f\left(x_{k}, y_{k}, z_{k}\right)\right)\right\|_{k} \leq \delta
$$

for all $\left(x_{1}, x_{2}, \ldots, x_{k}\right),\left(y_{1}, y_{2}, \ldots, y_{k}\right),\left(z_{1}, z_{2}, \ldots, z_{k}\right) \in E, a \in R$ and $\delta \geq 0$. Then there exists a unique additive mapping $A: E \rightarrow F$ such that

$$
A(x)=\lim _{n \rightarrow \infty} \frac{1}{3^{n}} f\left(3^{n} x+\frac{\left(3^{n}-1\right) a}{2}\right)
$$


and

$$
\sup _{k \in \mathbb{N}}\left\|f\left(x_{1}\right)-A\left(x_{1}\right), f\left(x_{2}\right)-A\left(x_{2}\right), \ldots, f\left(x_{k}\right)-A\left(x_{k}\right)\right\|_{k} \leq \frac{\delta}{2}
$$

for all $\left(x_{1}, x_{2}, \ldots, x_{k}\right) \in E$.

Proof. To prove this theorem we have to show the following steps:

(i) $\left\{f\left(3^{n} x+\frac{\left(3^{n}-1\right) a}{2}\right) / 3^{n}\right\}$ is a Cauchy sequence for every fixed $x \in E$.

(ii) There exists an additive mapping $A: E \rightarrow F$ defined by

$$
A(x)=\lim _{n \rightarrow \infty} \frac{1}{3^{n}} f\left(3^{n} x+\frac{\left(3^{n}-1\right) a}{2}\right)
$$

(iii) The mapping $A: E \rightarrow F$ satisfies

$$
\sup _{k \in \mathbb{N}}\left\|f\left(x_{1}\right)-A\left(x_{1}\right), f\left(x_{2}\right)-A\left(x_{2}\right), \ldots, f\left(x_{k}\right)-A\left(x_{k}\right)\right\|_{k} \leq \frac{\delta}{2}
$$

(iv) The mapping $A: E \rightarrow F$ is unique.

Let $x=y=z$ in $(3.1)$, we obtain

$$
\begin{aligned}
& \sup _{k \in \mathbb{N}}\left\|f\left(3 x_{1}+a\right)-3 f\left(x_{1}\right), f\left(3 x_{2}+a\right)-3 f\left(x_{2}\right), \ldots, f\left(3 x_{k}+a\right)-3 f\left(x_{k}\right)\right\|_{k} \leq \delta \\
& \sup _{k \in \mathbb{N}}\left\|\frac{f\left(3 x_{1}+a\right)}{3}-f\left(x_{1}\right), \frac{f\left(3 x_{2}+a\right)}{3}-f\left(x_{2}\right), \ldots, \frac{f\left(3 x_{k}+a\right)}{3}-f\left(x_{k}\right)\right\|_{k} \leq \frac{\delta}{3}
\end{aligned}
$$

Replacing $x$ by $3 x+a$ and dividing by 3 in (3.4) and also adding the resulting equation with (3.4), we get

$$
\begin{aligned}
& \sup _{k \in \mathbb{N}}\left\|\frac{f\left(9 x_{1}+4 a\right)}{3^{2}}-f\left(x_{1}\right), \frac{f\left(9 x_{2}+4 a\right)}{3^{2}}-f\left(x_{2}\right), \ldots, \frac{f\left(9 x_{k}+4 a\right)}{3^{2}}-f\left(x_{k}\right)\right\|_{k} \\
& \leq \frac{\delta}{3}\left(\frac{\delta}{3}+1\right)
\end{aligned}
$$

Taking induction on ' $n$ ', we have

$$
\sup _{k \in \mathbb{N}} \| \frac{f\left(3^{n} x_{1}+\frac{\left(3^{n}-1\right) a}{2}\right)}{3^{n}}-f\left(x_{1}\right), \frac{f\left(3^{n} x_{2}+\frac{\left(3^{n}-1\right) a}{2}\right)}{3^{n}}-f\left(x_{2}\right), \ldots,
$$




$$
\begin{aligned}
& \frac{f\left(3^{n} x_{k}+\frac{\left(3^{n}-1\right) a}{2}\right)}{3^{n}}-f\left(x_{k}\right) \|_{k} \leq \frac{\delta}{3} \sum_{i=0}^{n-1} \frac{1}{3^{i}} \\
& \sup _{k \in \mathbb{N}} \| \frac{f\left(3^{n} x_{1}+\frac{\left(3^{n}-1\right) a}{2}\right)}{3^{n}}-f\left(x_{1}\right), \frac{f\left(3^{n} x_{2}+\frac{\left(3^{n}-1\right) a}{2}\right)}{3^{n}}-f\left(x_{2}\right), \ldots, \\
& \frac{f\left(3^{n} x_{k}+\frac{\left(3^{n}-1\right) a}{2}\right)}{3^{n}}-f\left(x_{k}\right) \|_{k} \leq \frac{\delta}{3} \sum_{i=0}^{\infty} \frac{1}{3^{i}}
\end{aligned}
$$

for all $\left(x_{1}, x_{2}, \ldots, x_{k}\right) \in E$ and $n \geq 1$.

(i) Now to prove that the sequence $\left\{f\left(3^{n} x+\frac{\left(3^{n}-1\right) a}{2}\right) / 3^{n}\right\}$ is a Cauchy sequence, making $x \in E$ fix and replacing $\left(x_{1}, x_{2}, \ldots, x_{k}\right) \in E$ with $x, 3 x+$ $a, 3^{2} x+4 a, \ldots, 3^{k-1} x+\frac{\left(3^{k-1}-1\right) a}{2}$ in (3.5) and then using condition (N3) of definition (2.1), we have

$$
\begin{aligned}
\sup _{k \in \mathbb{N}} \| & \frac{f\left(3^{n} x_{1}+\frac{\left(3^{n}-1\right) a}{2}\right)}{3^{n}}-\frac{f\left(3^{m} x_{1}+\frac{\left(3^{m}-1\right) a}{2}\right)}{3^{m}}, \ldots, \\
& \frac{f\left(3^{n+k-1} x+\frac{\left(3^{n+k-1}-1\right) a}{2}\right)}{3^{n+k-1}}-\frac{f\left(3^{m+k-1} x+\frac{\left(3^{m+k-1}-1\right) a}{2}\right)}{3^{m+k-1}} \sum_{i=m}^{\infty} \frac{1}{3^{i}} \\
\sup _{k \in \mathbb{N}} \| & \frac{f\left(3^{n} x_{1}+\frac{\left(3^{n}-1\right) a}{2}\right)}{3^{n}}-\frac{f\left(3^{m} x_{1}+\frac{\left(3^{m}-1\right) a}{2}\right)}{3^{m}}, \ldots, \\
\leq & \frac{1}{3} \sum_{i=m}^{\infty} \frac{1}{3^{i}}\left[\frac{f\left(3^{n+k-1} x+\frac{\left(3^{n+k-1}-1\right) a}{2}\right)}{3^{n}}-\frac{f\left(3^{m+k-1} x+\frac{\left(3^{m+k-1}-1\right) a}{2}\right)}{3^{m}}\right] \|_{k}
\end{aligned}
$$

(ii) Since $F$ is Banach space, the sequence $\left\{f\left(3^{n} x+\frac{\left(3^{n}-1\right) a}{2}\right) / 3^{n}\right\}$ is a convergent sequence to a fixed point $A(x) \in F$ as $n \rightarrow \infty$, and hence a Cauchy sequence in $F$, therefore

$$
A(x)=\lim _{n \rightarrow \infty} \frac{1}{3^{n}} f\left(3^{n} x+\frac{\left(3^{n}-1\right) a}{2}\right)
$$


(iii) To prove that the additive mapping $A: E \rightarrow F$ satisfies the inequality (3.3) taking $n \rightarrow \infty$ in (3.5) and making the use of Lemma. 2.2, we obtain

$$
\sup _{k \in \mathbb{N}}\left\|f\left(x_{1}\right)-A\left(x_{1}\right), f\left(x_{2}\right)-A\left(x_{2}\right), \ldots, f\left(x_{k}\right)-A\left(x_{k}\right)\right\|_{k} \leq \frac{\delta}{2}
$$

(iv) For uniqueness of additive mapping $A$, let us consider another additive mapping $A^{1}: E \rightarrow F$, such that

$$
\begin{aligned}
\left\|A(x)-A^{1}(x)\right\|_{k} \leq & \left\|\frac{A\left(3^{n} x+\frac{\left(3^{n}-1\right) a}{2}\right)}{3^{n}}-\frac{A^{1}\left(3^{n} x+\frac{\left(3^{n}-1\right) a}{2}\right)}{3^{n}}\right\|_{k} \\
\leq & \left\|\frac{A\left(3^{n} x+\frac{\left(3^{n}-1\right) a}{2}\right)}{3^{n}}-\frac{f\left(3^{n} x+\frac{\left(3^{n}-1\right) a}{2}\right)}{3^{n}}\right\|_{k} \\
& \quad+\left\|\frac{f\left(3^{n} x+\frac{\left(3^{n}-1\right) a}{2}\right)}{3^{n}}-\frac{A^{1}\left(3^{n} x+\frac{\left(3^{n}-1\right) a}{2}\right)}{3^{n}}\right\|_{k} \\
\leq & \frac{\delta}{3^{n}}
\end{aligned}
$$

taking $n \rightarrow \infty$, we have

$$
\left\|A(x)-A^{1}(x)\right\|_{k} \leq 0 \quad \text { for all } x \in E
$$

which implies that

$$
A(x)=A^{1}(x) \quad \text { for all } x \in E
$$

This proves the required result.

Now, using the method of Proof of Theorem 3.1 and Proposition 3 by SoonMo Jung [15] and M.S. Moslehian and H.M. Srivastava [9] respectively, we prove the following results.

Proposition 3.2. Let $E$ be a normed space and let $\left(F^{n},\|\cdot\|_{n}\right), n \in \mathbb{N}$ be a multi-Banach space. Let $f: E \rightarrow F$ be a mapping satisfying the following inequality with $\delta, \epsilon \geq 0$,

$$
\sup _{k \in \mathbb{N}}\left\|D_{a}\left(f\left(x_{1}, y_{1}, z_{1}\right)\right), D_{a}\left(f\left(x_{2}, y_{2}, z_{2}\right)\right), \ldots, D_{a}\left(f\left(x_{k}, y_{k}, z_{k}\right)\right)\right\|_{k} \leq \delta
$$

for all $\left(x_{1}, x_{2}, \ldots, x_{k}\right),\left(y_{1}, y_{2}, \ldots, y_{k}\right),\left(z_{1}, z_{2}, \ldots, z_{k}\right) \in E$ with the condition

$$
\left\|\left(x_{1}, x_{2}, \ldots, x_{k}\right)\right\|+\left\|\left(y_{1}, y_{2}, \ldots, y_{k}\right)\right\|+\left\|\left(z_{1}, z_{2}, \ldots, z_{k}\right)\right\| \geq \epsilon
$$


Then there exists a unique additive mapping $A: E \rightarrow F$ such that

$$
\sup _{k \in \mathbb{N}}\left\|\left(f\left(x_{1}\right)-A\left(x_{1}\right), f\left(x_{2}\right)-A\left(x_{2}\right), \ldots, f\left(x_{k}\right)-A\left(x_{k}\right)\right)\right\|_{k} \leq 7 \delta
$$

for all $\left(x_{1}, x_{2}, \ldots, x_{k}\right) \in E$.

Proof. For the case $\|x\|+\|y\|+\|z\| \geq \delta$, the result holds obviously. Therefore, let us consider the case for $\|x\|+\|y\|+\|z\|<\delta$, where $x=\left(x_{1}, x_{2}, \ldots, x_{k}\right), y=$ $\left(y_{1}, y_{2}, \ldots, y_{k}\right)$ and $z=\left(z_{1}, z_{2}, \ldots, z_{k}\right)$. If $x=y=z=0$, there exists an element $w=\left(w_{1}, w_{2}, \ldots, w_{k}\right) \in E^{k}$ with $\|w\|=d$.

Now, for $x \neq 0, y \neq 0$ and $z \neq 0$, let

$$
w= \begin{cases}x+\frac{\delta x}{\|x\|_{k}} & \text { if }\|x\|_{k} \geq \max \left(\|y\|_{k},\|z\|_{k}\right) \\ y+\frac{\delta y}{\|y\|_{k}} & \text { if }\|y\|_{k}>\max \left(\|x\|_{k},\|z\|_{k}\right) \\ z+\frac{\delta z}{\|z\|_{k}} & \text { if }\|z\|_{k}>\max \left(\|y\|_{k},\|x\|_{k}\right)\end{cases}
$$

Then,

$$
\begin{aligned}
& \|x\|+\|y-w\|+\|z+w\| \geq \delta \\
& \|x-w\|+\|y+w\|+\|w\| \geq \delta \\
& \|x-w\|+\|y\|+\|2 w\| \geq \delta \\
& \|x+w\|+\|z-w\|+\|2 w\| \geq \delta \\
& \|x+w\|+\|z\|+\|w\| \geq \delta \\
& \|y-w\|+\|z+w\|+\|w\| \geq \delta \\
& \|y+w\|+\|z-w\|+\|w\| \geq \delta
\end{aligned}
$$

Now, using the inequalities of (3.11) in (3.7), we obtain

$$
\begin{aligned}
& \| f\left(x_{1}+y_{1}+z_{1}+a\right)-f\left(x_{1}\right)-f\left(y_{1}\right)-f\left(z_{1}\right), \ldots, \\
& \quad f\left(x_{k}+y_{k}+z_{k}+a\right)-f\left(x_{k}\right)-f\left(y_{k}\right)-f\left(z_{k}\right) \|_{k} \\
& \leq \|\left(f\left(x_{1}+y_{1}+z_{1}+a\right)-f\left(x_{1}\right)-f\left(y_{1}-w_{1}\right)-f\left(z_{1}+w_{1}\right), \ldots,\right. \\
& \left.\quad f\left(x_{k}+y_{k}+z_{k}+a\right)-f\left(x_{k}\right)-f\left(y_{k}-w_{k}\right)-f\left(z_{k}+w_{k}\right)\right) \\
& \quad-\left(\left(f\left(x_{1}+y_{1}+w_{1}+a\right)-f\left(x_{1}-w_{1}\right)-f\left(y_{1}+w_{1}\right)-f\left(w_{1}\right), \ldots\right.\right. \\
& \quad\left(f\left(x_{k}+y_{k}+w_{k}+a\right)-f\left(x_{k}-w_{k}\right)-f\left(y_{k}+w_{k}\right)-f\left(w_{k}\right)\right) \\
& \quad+\left(\left(f\left(x_{1}+y_{1}+w_{1}+a\right)-f\left(x_{1}-w_{1}\right)-f\left(y_{1}\right)-f\left(2 w_{1}\right), \ldots\right.\right.
\end{aligned}
$$




$$
\begin{aligned}
& \left(f\left(x_{k}+y_{k}+w_{k}+a\right)-f\left(x_{k}-w_{k}\right)-f\left(y_{k}\right)-f\left(2 w_{k}\right)\right) \\
- & \left(\left(f\left(x_{1}+z_{1}+2 w_{1}+a\right)-f\left(x_{1}+w_{1}\right)-f\left(z_{1}-w_{1}\right)-f\left(2 w_{1}\right), \ldots\right.\right. \\
& \left(f\left(x_{k}+z_{k}+2 w_{k}+a\right)-f\left(x_{k}+w_{k}\right)-f\left(z_{k}-w_{k}\right)-f\left(2 w_{k}\right)\right) \\
+ & \left(\left(f\left(x_{1}+z_{1}+2 w_{1}+a\right)-f\left(x_{1}+w_{1}\right)-f\left(z_{1}\right)-f\left(w_{1}\right), \ldots\right.\right. \\
& \left(f\left(x_{k}+z_{k}+2 w_{k}+a\right)-f\left(x_{k}+w_{k}\right)-f\left(z_{k}\right)-f\left(w_{k}\right)\right) \\
- & \left(\left(f\left(y_{1}+z_{1}+w_{1}+a\right)-f\left(y_{1}-w_{1}\right)-f\left(z_{1}+w_{1}\right)-f\left(w_{1}\right), \ldots\right.\right. \\
& \left(f\left(y_{k}+z_{k}+w_{k}+a\right)-f\left(y_{k}-w_{k}\right)-f\left(z_{k}+w_{k}\right)-f\left(w_{k}\right)\right) \\
+ & \left(\left(f\left(y_{1}+z_{1}+w_{1}+a\right)-f\left(y_{1}+w_{1}\right)-f\left(z_{1}-w_{1}\right)-f\left(w_{1}\right), \ldots\right.\right. \\
& \left(f\left(y_{k}+z_{k}+w_{k}+a\right)-f\left(y_{k}+w_{k}\right)-f\left(z_{k}-w_{k}\right)-f\left(w_{k}\right)\right) \|_{k}
\end{aligned}
$$

Thus, we obtain

$$
\begin{aligned}
& \| f\left(x_{1}+y_{1}+z_{1}+a\right)-f\left(x_{1}\right)-f\left(y_{1}\right)-f\left(z_{1}\right), \ldots \\
& \quad f\left(x_{k}+y_{k}+z_{k}+a\right)-f\left(x_{k}\right)-f\left(y_{k}\right)-f\left(z_{k}\right) \|_{k} \leq 7 \delta
\end{aligned}
$$

for all $\left(x_{1}, x_{2}, \ldots, x_{k}\right),\left(y_{1}, y_{2}, \ldots, y_{k}\right),\left(z_{1}, z_{2}, \ldots, z_{k}\right) \in E$.

Thus, from the above proposition we have deduced the following corollary. Let us consider the domain

$$
D=\left\{(x, y, z) \in E^{k} \times E^{k} \times E^{k}:\|x\|_{k}+\|y\|_{k}+\|z\|_{k}<\delta\right\}
$$

For non-negative real number $\delta$. Then, using above proposition the result holds on the domain $E^{k} \times E^{k} \times E^{k} D$ with the condition $\|x\|_{k}+\|y\|_{k}+\|z\|_{k} \geq 3 \delta$.

Corollary 3.3. Let $E$ be a normed space and let $\left(F^{n},\|\cdot\|_{n}\right), n \in \mathbb{N}$ be a multi-Banach space. Let $f: E \rightarrow F$ be a mapping satisfying the following inequality with $\delta, \epsilon \geq 0$

$$
\sup _{k \in \mathbb{N}}\left\|D_{a}\left(f\left(x_{1}, y_{1}, z_{1}\right)\right), D_{a}\left(f\left(x_{2}, y_{2}, z_{2}\right)\right), \ldots, D_{a}\left(f\left(x_{k}, y_{k}, z_{k}\right)\right)\right\|_{k} \leq \delta
$$

for all $k>0$ and $\left(x_{1}, x_{2}, \ldots, x_{k}\right),\left(y_{1}, y_{2}, \ldots, y_{k}\right),\left(z_{1}, z_{2}, \ldots, z_{k}\right) \in E^{k} \times E^{k} \times$ $E^{k} D$ with the condition

$$
\left\|\left(x_{1}, x_{2}, \ldots, x_{k}\right)\right\|+\left\|\left(y_{1}, y_{2}, \ldots, y_{k}\right)\right\|+\left\|\left(z_{1}, z_{2}, \ldots, z_{k}\right)\right\| \geq \epsilon
$$

Then there exists a unique additive mapping $A: E \rightarrow F$ such that

$$
\sup _{k \in N}\left\|\left(f\left(x_{1}\right)-A\left(x_{1}\right), f\left(x_{2}\right)-A\left(x_{2}\right), \ldots, f\left(x_{k}\right)-A\left(x_{k}\right)\right)\right\|_{k} \leq 7 \delta
$$

for all $\left(x_{1}, x_{2}, \ldots, x_{k}\right) \in E$. 


\section{References}

[1] D.H. Hyers, On the Stability of the Linear Functional Equation, Proc. Nat. Acad. Sci. U.S.A. (27) (1941), pp. 222-224.

[2] F. Skof, Local properties and approximations of operators, Rend. Sem. Mat. Fis. Milano, (53) (1983) pp.113-129.

[3] H.G. Dales and M.E. Polyakov, Multi-normed spaces and multi-Banach algebras, preprint.

[4] H.G. Dales and M.S. Moslehian, Stability of mappings on multi-normed spaces, Glasgow Mathematical Journal, (49) (2) (2007), pp. 321-332.

[5] J.R. Lee and C. Park, Stability of the Cauchy Functional Equations in Banach Algebras, Korean J. Math. (17) (1) (2009), pp. 91-102.

[6] L. Wang, B. Liu and R. Bai, Stability of a Mixed Type Functional Equation on Multi-Banach Spaces: A Fixed Point Approach, Fixed Point Theory and Applications, vol. 2010, Art. ID 283827, 9 pages.

[7] M.S. Moslehian, Superstability of higher derivations in multi-Banach algebras, Tamsui Oxford J. of Math. Sci., (24) (4) (2008), pp. 417-427.

[8] M.S. Moslehian, K. Nikodem, and D. Popa, Asymptotic aspect of the quadratic functional equation in multi-normed spaces, J. of Math. Anal. Appl., (355) (2) (2009), pp. 717-724.

[9] M.S. Moslehian and H.M. Srivastava, Jensens functional equations in multi-normed spaces, Taiwnese J. of Math., (14) (2) (2010), pp. 453-462.

[10] M. Kumar and Ashish, Stability of Functional Equations in Multi-Banach Space via Fixed Point Approach, Int. J. Com. Appl., (44) (7) (2012), pp. $35-40$.

[11] M. Kumar and Ashish, Stability of Jensen Type Quadratic Functional equations in multi-Banach spaces, Int. J. of Math. Arch., (3) (4) (2012), pp.1372-137.

[12] P.W. Cholewa, Remarks on the stability of functional equations, Aequations Math. (27) (1984), pp. 76-86.

[13] P. Gavruta, A Generalization of the Hyers-Ulam-Rassias Stability of Approximately Additive Mappings, J. Math. Anal. Appl., (184) (1994), pp. 431-436. 
[14] R. Chugh and Ashish, On the stability of generalized Cauchy linear functional equations, Int. J. of Math. Anal., (6) (29) (2012), pp. 1403-1413.

[15] S.M. Jung, Hyers-Ulam-Rassias Stability of Jensen's Equation and its Application, Proc. Amer. Math. Soc., (126) (1998), pp. 3137-3143.

[16] S.M. Ulam, A Collection of the Mathematical Problems, Interscience Publ., New York, 1960.

[17] S. Czerwik, Functional Equations and Inequalities in Several Variables, World Scientific Publ. Co., New Jersey, London, Singapore and Hong Kong, 2002.

[18] S. Czerwik, Stability of Functional Equations of Ulam-Hyers-Rassias Type, Hadronic Press Inc., Palm Harbor, Florida, 2003.

[19] T. Aoki, On the stability of the linear transformation in Banach spaces, $J$. of Math. Soc., (2) (1-2) (1950), pp. 64-66.

[20] Th. M. Rassias, On the stability of the Linear mapping in Banach spaces, Proc. of Amer. Math. Soc., (72) (2) (1978), pp. 297-300.

[21] Th. M. Rassias, On the stability of the functional equations in Banach spaces, J. Math. Anal. Appl., (251) (2000), pp. 264-284.

[22] Z. Wang, X. Li and Th. M. Rassias, Stability of an Additive-Cubic-Quartic Functional Equation in multi-Banach Spaces, Abstract and Applied Analysis, (2011) (2011), Article ID 536520, 11 pages. 
